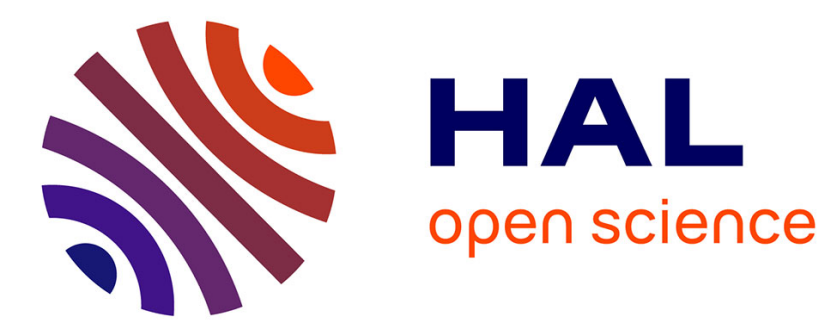

\title{
Non-isothermal Compositional Two-Phase Darcy Flow: Formulation and Outflow Boundary Condition
}

\author{
Laurence Beaude, Konstantin Brenner, Simon Lopez, Roland Masson, Farid F
}

Smaï

\section{- To cite this version:}

Laurence Beaude, Konstantin Brenner, Simon Lopez, Roland Masson, Farid F Smaï. Non-isothermal Compositional Two-Phase Darcy Flow: Formulation and Outflow Boundary Condition. FVCA 2017

- International Conference on Finite Volumes for Complex Applications VIII, Jun 2017, Lille, France. 10.1007/978-3-319-57394-6_34. hal-01541151

\section{HAL Id: hal-01541151 \\ https://hal.science/hal-01541151}

Submitted on 25 Jun 2017

HAL is a multi-disciplinary open access archive for the deposit and dissemination of scientific research documents, whether they are published or not. The documents may come from teaching and research institutions in France or abroad, or from public or private research centers.
L'archive ouverte pluridisciplinaire HAL, est destinée au dépôt et à la diffusion de documents scientifiques de niveau recherche, publiés ou non, émanant des établissements d'enseignement et de recherche français ou étrangers, des laboratoires publics ou privés. 


\title{
Non-isothermal compositional two-phase Darcy flow: formulation and outflow boundary condition
}

\author{
L. Beaude, K. Brenner, S. Lopez, R. Masson, F. Smai
}

\begin{abstract}
This article deals with the modelling and formulation of compositional gas liquid Darcy flow. Our model includes an advanced boundary condition at the interface between the porous medium and the atmosphere accounting for convective mass and energy transfer, liquid evaporation, and liquid outflow. The formulation is based on a fixed set of unknowns whatever the set of present phases. The thermodynamical equilibrium is expressed as complementary constraints. The model and its formulation are applied to the simulation of the Bouillante high energy geothermal field in Guadeloupe characterized by a high temperature closed to the surface.
\end{abstract}

Key words: Non-isothermal compositional two-phase Darcy flow model, geothermal energy, boundary conditions for the interaction ground-atmosphere, finite volume scheme

\section{Non-isothermal compositional two-phase Darcy flow model}

We consider a non-isothermal compositional liquid gas Darcy flow model with $\mathscr{P}=\{g, l\}$ denoting the set of gas and liquid phases. The set of components is denoted by $\mathscr{C}$ including typically a water component which can vaporize in the gas phase and a set of gaseous components which can dissolve in the liquid phase. The thermodynamical properties of each phase $\alpha \in \mathscr{P}$ depend on its pressure $P^{\alpha}$, the temperature $T$ and its molar fractions $C^{\alpha}=\left(C_{i}^{\alpha}\right)_{i \in \mathscr{C}}$.

\footnotetext{
Laurence Beaude, Konstantin Brenner, Roland Masson

Université Côte d'Azur, Inria, CNRS, LJAD,

UMR 7351 CNRS, Parc Valrose 06108 Nice Cedex 02, France,

e-mail: laurence.beaude,konstantin.brenner,roland.masson@unice.fr

Simon Lopez, Farid Smai

BRGM Orléans France,

3 avenue Claude-Guillemin, BP 36009, 45060 Orléans Cedex 2, France,

e-mail: s.lopez,f.smai@brgm.fr
} 
For each phase $\alpha \in \mathscr{P}$, we denote by $\zeta^{\alpha}\left(P^{\alpha}, T, C^{\alpha}\right)$ its molar density, by $\rho^{\alpha}\left(P^{\alpha}, T, C^{\alpha}\right)$ its mass density, by $\mu^{\alpha}\left(P^{\alpha}, T, C^{\alpha}\right)$ its dynamic viscosity, by $e^{\alpha}\left(P^{\alpha}, T, C^{\alpha}\right)$ its molar internal energy and by $h^{\alpha}\left(P^{\alpha}, T, C^{\alpha}\right)$ its molar enthalpy. Thermodynamical equilibrium between the gas and liquid phases will be assumed for each component and governed by the fugacity functions denoted by $f^{\alpha}\left(P^{\alpha}, T, C^{\alpha}\right)=$ $\left(f_{i}^{\alpha}\left(P^{\alpha}, T, C^{\alpha}\right)\right)_{i \in \mathscr{C}}$.

The rock porosity is denoted by $\phi(\mathbf{x})$ and the rock permeability tensor by $\mathbf{K}(\mathbf{x})$ where $\mathbf{x}$ denotes the spatial coordinates. The hydrodynamical Darcy laws are characterized by the relative permeabilities $k_{r}^{\alpha}\left(S^{\alpha}\right)$, function of the phase saturation $S^{\alpha}$ for each phase $\alpha \in \mathscr{P}$, and by the capillary pressure $P_{c}\left(S^{g}\right)=P^{g}-P^{l}$.

Our formulation of the model is based on the fixed set of unknowns defined by

$$
X=\left(P^{\alpha}, T, S^{\alpha}, C^{\alpha}, \alpha \in \mathscr{P}\right) .
$$

Let $n_{i}(X)$ be the number of moles of the component $i \in \mathscr{C}$ per unit pore volume defined as

$$
n_{i}(X)=\sum_{\alpha \in \mathscr{P}} \zeta^{\alpha} S^{\alpha} C_{i}^{\alpha}, \quad i \in \mathscr{C} .
$$

We introduce the rock energy per unit rock volume defined by $E_{r}\left(P^{\alpha}, T\right)$ and the fluid energy per unit pore volume defined by

$$
E(X)=\sum_{\alpha \in \mathscr{P}} \zeta^{\alpha} S^{\alpha} e^{\alpha}
$$

Let us denote by $\mathbf{g}$ the gravitational acceleration vector. The Darcy velocity of the phase $\alpha \in \mathscr{P}$ is then given by

$$
\mathbf{V}^{\alpha}=-\frac{k_{r}^{\alpha}}{\mu^{\alpha}} \mathbf{K}(\mathbf{x})\left(\nabla P^{\alpha}-\rho^{\alpha} \mathbf{g}\right)
$$

The total molar flux of the component $i \in \mathscr{C}$ is denoted by $\mathbf{q}_{i}$ and the energy flux by $\mathbf{q}_{e}$, with

$$
\mathbf{q}_{i}=\sum_{\alpha \in \mathscr{P}} C_{i}^{\alpha} \zeta^{\alpha} \mathbf{V}^{\alpha}, \quad \mathbf{q}_{e}=\sum_{\alpha \in \mathscr{P}} h^{\alpha} \zeta^{\alpha} \mathbf{V}^{\alpha}-\lambda \nabla T
$$

where $\lambda$ stands for the thermal conductivity of the fluid and rock mixture.

The model accounts for the molar conservation of each component $i \in \mathscr{C}$ together with the energy conservation

$$
\begin{gathered}
\phi(\mathbf{x}) \partial_{t} n_{i}+\operatorname{div}\left(\mathbf{q}_{i}\right)=0, \quad i \in \mathscr{C}, \\
\phi(\mathbf{x}) \partial_{t} E+(1-\phi(\mathbf{x})) \partial_{t} E_{r}+\operatorname{div}\left(\mathbf{q}_{e}\right)=0 .
\end{gathered}
$$

It is complemented by the following capillary relation between the two phase pressures and the pore volume balance 


$$
\left\{\begin{array}{l}
P_{c}\left(S^{g}\right)=P^{g}-P^{l}, \\
\sum_{\alpha \in \mathscr{P}} S^{\alpha}=1 .
\end{array}\right.
$$

Due to change of phase reactions assumed to be at equilibrium, phases can appear or disappear. In our formulation the molar fractions $C^{\alpha}$ of an absent phase $\alpha$ are extended by the ones at equilibrium with the present phase. It results that the thermodynamical equilibrium can be expressed as the following complementary constraints for each phase $\alpha \in \mathscr{P}$ combined with the equality of the gas and liquid fugacities of each component [5]

$$
\left\{\begin{array}{l}
S^{\alpha}\left(1-\sum_{i \in \mathscr{C}} C_{i}^{\alpha}\right)=0, \quad \alpha \in \mathscr{P}, \\
S^{\alpha} \geq 0, \quad 1-\sum_{i \in \mathscr{C}} C_{i}^{\alpha} \geq 0, \\
f_{i}^{g}\left(P^{g}, T, C^{g}\right)=f_{i}^{l}\left(P^{l}, T, C^{l}\right), \quad i \in \mathscr{C} .
\end{array}\right.
$$

Note that our formulation of the model leads to a fix set of unknowns and equations which is independent of the set of present phases and expresses the thermodynamical equilibrium as complementary constraints. This will allow the use of non-smooth Newton methods to solve the non-linear systems at each time step of the simulation as specified in the numerical section.

\section{Boundary condition at the interface between the porous medium and the atmosphere}

The fluid and energy transport in high energy geothermal systems is deeply governed by the conditions set at the boundary of the computational domain. In particular, it is well known that the modelling of the interaction between the porous medium model and the atmosphere plays an important role [6]. In this section we propose a boundary condition model taking into account the convective molar and energy transfer and the vaporization of the liquid phase in the atmosphere as well as a liquid outflow condition.

The convective molar and energy boundary layers induced by the turbulent gas flow in the atmosphere are expressed using two boundary layer thicknesses denoted by $\delta_{m}$ for the molar convective transfer and by $\delta_{T}$ for the energy convective transfer. Let us also introduce the additional unknown $q^{g, a t m}$ accounting for the gas molar flow rate at the interface on the atmosphere side oriented outward from the porous medium domain. The liquid phase is assumed to vaporize instantaneously when leaving the porous medium as long as the atmosphere is not saturated with water vapour. As soon as the atmosphere is vapour saturated at the interface, a liquid molar flow rate $q^{l, a t m}$ is allowed to exit the porous medium. The prescribed far field atmospheric conditions are defined by the gas molar fractions $C_{\infty}^{g, a t m}$, the temperature $T_{\infty}^{a t m}$ and the gas pressure $P^{a t m}$. The model assumes the continuity of the gas phase characterized by the continuity of the gas pressure $P^{g}=P^{a t m}$, of the temperature $T$ 
and of the gas molar fractions $C^{g}$ at the interface. Let us recall that $P^{l}$ is the liquid pressure and $C^{l}$ the liquid molar fractions at the interface on the porous medium side. We introduce the liquid molar fractions $C^{l, a t m}=\left(C_{i}^{l, a t m}\right)_{i \in \mathscr{C}}$ at the interface on the atmosphere side by the one at thermodynamical equilibrium with the gas phase. It is obtained by the equation $f^{l}\left(P^{a t m}, T, C^{l, a t m}\right)=f^{g}\left(P^{g}, T, C^{g}\right)$. Note that, due to the jump of the capillary pressure which vanishes on the atmosphere side, $C^{l, \text { atm }}$ does not match in general with $C^{l}$ which satisfies $f^{l}\left(P^{l}, T, C^{l}\right)=f^{g}\left(P^{g}, T, C^{g}\right)$.

Let us denote by $(u)^{+}$(resp. $\left.(u)^{-}\right)$the positive part (the negative part) of the variable $u$ such that $(u)^{+}=\max (0, u)\left(\right.$ resp. $(u)^{-}=\max (0,-u)$ ).

At the interface, on the atmosphere side, the component gas molar normal flux $q_{i}^{g, a t m}, i \in \mathscr{C}$ and the gas energy normal flux $q_{e}^{g, a t m}$ are defined by

$$
\begin{gathered}
q_{i}^{g, a t m}=\left(q^{g, a t m}\right)^{+} C_{i}^{g}-\left(q^{g, a t m}\right)^{-} C_{i, \infty}^{g, a t m}+\frac{\zeta^{g} D^{g}}{\delta_{m}}\left(C_{i}^{g}-C_{i, \infty}^{g, a t m}\right), \quad i \in \mathscr{C}, \\
q_{e}^{g, a t m}=\left(q^{g, a t m}\right)^{+} h^{g}\left(P^{g}, T, C^{g}\right)-\left(q^{g, a t m}\right)^{-} h_{\infty}^{g, a t m}+\frac{\lambda^{g}}{\delta_{T}}\left(T-T_{\infty}^{a t m}\right),
\end{gathered}
$$

where $D^{g}$ is the gas molecular diffusion coefficient, $\lambda^{g}$ is the gas thermal conductivity and $h_{\infty}^{g, a t m}=h^{g}\left(P^{a t m}, T_{\infty}^{a t m}, C_{\infty}^{g, a t m}\right)$ is the far field atmospheric gas enthalpy.

The model prescribes the continuity at the interface of the molar and energy normal fluxes:

$$
\left\{\begin{array}{l}
\mathbf{q}_{i} \cdot \mathbf{n}=q_{i}^{g, a t m}+C_{i}^{l, a t m} q^{l, a t m}, \quad i \in \mathscr{C}, \\
\mathbf{q}_{e} \cdot \mathbf{n}=q_{e}^{g, a t m}+h^{l}\left(P^{g}, T, C^{l, a t m}\right) q^{l, a t m},
\end{array}\right.
$$

where the unit normal vector $\mathbf{n}$ at the interface is oriented outward from the porous medium domain.

The liquid molar overflow rate $q^{l, a t m}$ is determined by the following complementary constraints accounting for the thermodynamical equilibrium between the liquid and gas phases at the interface in the atmosphere:

$$
\left\{\begin{array}{l}
\left(1-\sum_{i \in \mathscr{C}} C_{i}^{l, a t m}\right) q^{l, a t m}=0, \\
1-\sum_{i \in \mathscr{C}} C_{i}^{l, a t m} \geq 0, \quad q^{l, a t m} \geq 0 .
\end{array}\right.
$$

It remains to eliminate the liquid molar fractions $C^{l, a t m}$ from equations (6) and (7). Following [4], let us consider for $f \in \mathbb{R}^{\mathscr{C}}$ the function $\mathcal{C}^{l}\left(f, P^{l}, T\right) \in \mathbb{R}^{\mathscr{C}}$ defined as the unique solution of the equation $f^{l}\left(P^{l}, T, C^{l}\right)=f$.

From $f^{g}\left(P^{g}, T, C^{g}\right)=f^{l}\left(P^{g}, T, C^{l, a t m}\right)=f^{l}\left(P^{l}, T, C^{l}\right)$ it results that

$$
C^{l, a t m}=\mathrm{e}^{l}\left(f^{l}\left(P^{l}, T, C^{l}\right), P^{g}, T\right) .
$$

On the one hand, if $S^{l}>0$, it follows that 


$$
\begin{aligned}
1-\sum_{i \in \mathscr{C}} C_{i}^{l, a t m} & =\sum_{i \in \mathscr{C}}\left(C_{i}^{l}-C_{i}^{l, a t m}\right) \\
& =\sum_{i \in \mathscr{C}}\left(\mathcal{C}_{i}^{l}\left(f^{l}\left(P^{l}, T, C^{l}\right), P^{l}, T\right)-\mathcal{C}_{i}^{l}\left(f^{l}\left(P^{l}, T, C^{l}\right), P^{g}, T\right)\right) .
\end{aligned}
$$

Following [5], we can assume that the function $\sum_{i \in \mathscr{C}} \mathcal{C}_{i}^{l}(f, P, T)$ is strictly decreasing with respect to $P$, it results that the complementary constraints (7) is equivalent to

$$
\left\{\begin{array}{l}
\left(P^{g}-P^{l}\right) q^{l, a t m}=0 \\
P^{g}-P^{l} \geq 0, q^{l, a t m} \geq 0
\end{array}\right.
$$

On the other hand, if $S^{l}=0$ then one has $P^{g}-P^{l}=P_{c}(1)>0$ and $\sum_{i \in \mathscr{C}} C_{i}^{l, a t m}<1$. It results that both conditions (9) and (7) imply that $q^{l, a t m}=0$. Finally, let us remark that (9) and $C^{l}=\mathrm{e}^{l}\left(f^{l}\left(P^{l}, T, C^{l}\right), P^{l}, T\right)$ imply that $C^{l, a t m}$ can be replaced by $C^{l}$ in the normal flux continuity equations (6).

In order to account for a non zero entry pressure for the capillary function $P_{c}\left(S^{g}\right)$, let us choose $P_{c}$ as primary unknown rather than $S^{g}$ and denote by $\mathcal{S}^{g}\left(P_{c}\right)$ the inverse of the monotone graph extension of the capillary pressure. As detailed in [2], a switch of variable between $S^{g}$ and $P_{c}$ could also be used in order to account for non invertible capillary functions.

To conclude, our evaporation - overflow boundary condition model is defined at the interface by the set of unknowns $X_{\Gamma}=\left(q^{g, a t m}, q^{l, a t m}, T, P^{\alpha}, S^{\alpha}, C^{\alpha}, \alpha \in \mathscr{P}\right)$ and the set of equations:

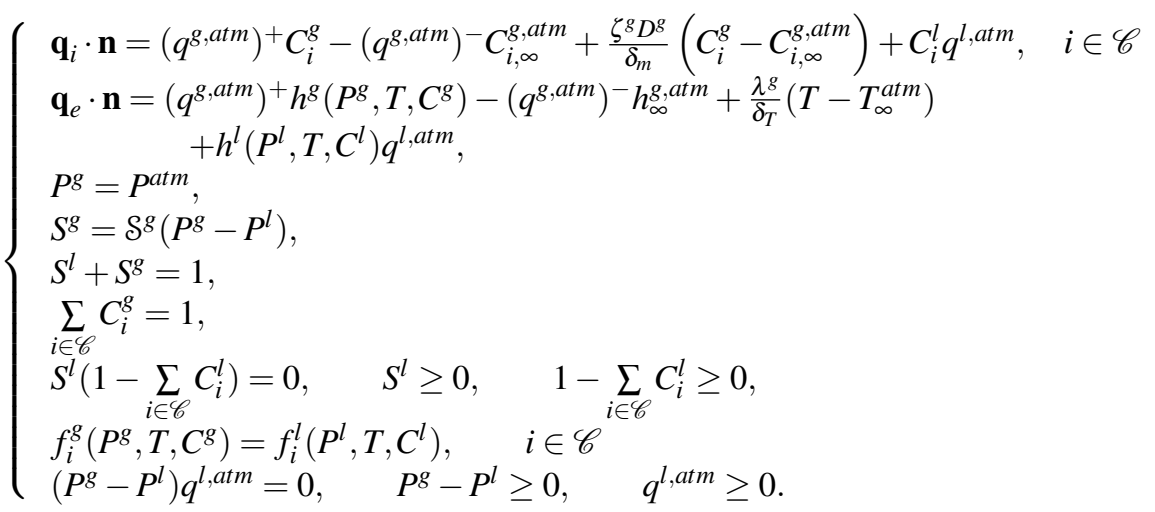

\section{Numerical tests}

The system of equations is discretized using a fully implicit Euler scheme in time and a finite volume discretization in space with a Two Point Flux Approximation (TPFA) [3]. The mobility terms of each phase are upwinded with respect to the sign of the phase Darcy flux. The non linear system is solved at each time step by a semi- 
smooth Newton algorithm (Newton-Min) adapted to complementary constraints [1]. In order to reduce the size of the linear systems to $\# \mathscr{C}+1$ equations and unknowns in each degrees of freedom (the cells and boundary faces), the set of unknowns is splitted into $\# \mathscr{C}+1$ primary unknowns and remaining secondary unknowns. This splitting is done for each degree of freedom in such a way that the Jacobian of the local closure laws with respect to the secondary unknowns is non singular. Note that the non linear convergence criterion is prescribed on the maximum of the relative norms of the energy balance equation residual and of each component mole balance equation residual. This relative norm is defined as the ratio of the residual $l 1$-norm by the initial residual $l 1$-norm.

Table 1 Choices of the primary unknowns depending on the complementary constraints.

\begin{tabular}{lccc}
\hline \multicolumn{2}{c}{ Evaporation - overflow boundary } & \multicolumn{2}{c}{ Interior cell and other boundaries } \\
\hline$q^{l, \text { atm }}<P^{g}-P^{l}$ & $q^{g, a t m}, P_{c},\left(C_{i}^{l}\right)_{i=1, \# \mathscr{C}-1}$ & $1-\sum_{i \in \mathscr{C}} C_{i}^{g}<S^{g}$ & $P^{g}, S^{g},\left(C_{i}^{l}\right)_{i=1, \# \mathscr{C}-1}$ \\
$1-\sum_{i \in \mathscr{C}} C_{i}^{l}<S^{l}$ & $1-\sum_{i \in \mathscr{C}} C_{i}^{l}<S^{l}$ & \\
\hline$P^{g}-P^{l}<q^{l, a t m}$ & $q^{g, a t m}, q^{l, a t m}, T,\left(C_{i}^{l}\right)_{i=1, \# \mathscr{C}-2}$ & $S^{g}<1-\sum_{i \in \mathscr{C}} C_{i}^{g}$ & $P^{g}, T,\left(C_{i}^{l}\right)_{i=1, \# \mathscr{C}-1}$ \\
$1-\sum_{i \in \mathscr{C}} C_{i}^{l}<S^{l}$ & & $1-\sum_{i \in \mathscr{C}} C_{i}^{l}<S^{l}$ & \\
\hline$q^{l, a t m}<P^{g}-P^{l}$ & $q^{g, a t m}, T,\left(C_{i}^{g}\right)_{i=1, \# \mathscr{C}-1}$ & $1-\sum_{i \in \mathscr{C}} C_{i}^{g}<S^{g}$ & $P^{g}, T,\left(C_{i}^{g}\right)_{i=1, \# \mathscr{C}-1}$ \\
$S^{l}<1-\sum_{i \in \mathscr{C}} C_{i}^{l}$ & & $S^{l}<1-\sum_{i \in \mathscr{C}} C_{i}^{l}$ & \\
\hline
\end{tabular}

The impact of the boundary condition is studied over a 2D dimensional test case representing a simplified domain of the Bouillante geothermal reservoir. A Voronoi mesh satisfying the admissibility condition of TPFA schemes at both inner and boundary faces is used [3]. We consider an homogeneous porous medium of porosity $\phi(\mathbf{x})=0.35$ and isotropic permeability $\mathbf{K}(\mathbf{x})=K * I$ with $K=1 D$. The relative permeabilities are defined as $k_{r}^{\alpha}\left(S^{\alpha}\right)=\left(S^{\alpha}\right)^{2}$ for each phase $\alpha \in \mathscr{P}$. The capillary pressure function is given by the Corey law $P_{c}\left(S^{g}\right)=-b \ln \left(1-S^{g}\right)$ for $S^{g} \in\left[0, s_{1}\right]$ and by $P_{c}\left(S^{g}\right)=-b \ln \left(1-s_{1}\right)+\frac{b}{1-s_{1}}\left(S^{g}-s_{1}\right)$ for $S^{g} \in\left(s_{1}, 1\right]$ with $b=210^{5} \mathrm{~Pa}$ and $s_{1}=0.99$. The capillary pressure is regularized to allow the disappearance of the liquid phase. The liquid and gas phases are a mixture of two components, the water denoted by $w$ and the air denoted by $a$.

The gas thermodynamical laws are defined by the perfect gas molar density $\zeta^{g}=$ $\frac{P^{g}}{R T}$, with $R=8.314 J . K^{-1} . \mathrm{mol}^{-1}$ and the viscosity $\mu^{g}=(0.361 T-10.2) 10^{-7}$ Pa.s. The liquid molar density and viscosity as well as the liquid and gas enthalpies are taken from [7]. The vapour pressure $P_{\text {sat }}(T)$ is given by the Clausius-Clapeyron equation and the Henry constant of the air component is set to $H_{a}=10^{8} \mathrm{~Pa}$. The molar internal energy of each phase is considered to be equal to its enthalpy. Finally, the fugacities are defined by 


$$
\left\{\begin{array}{l}
f_{i}^{g}=C_{i}^{g} P^{g}, \quad i=a, w, \\
f_{a}^{l}=C_{a}^{l} H_{a}, \\
f_{w}^{l}=C_{w}^{l} P_{\text {sat }}(T) \exp \left(-\frac{P_{\text {sat }}(T)-P^{l}}{1000 R T / 0.018}\right) .
\end{array}\right.
$$

The simulation is run over 400 years, with an initial time step of 5 days and a maximum time step of 700 days. The mesh contains approximatively 3000 cells and is refined at the neighbourhood of the top boundary with a volume ratio of 29 between the smallest and the largest cells of the mesh.

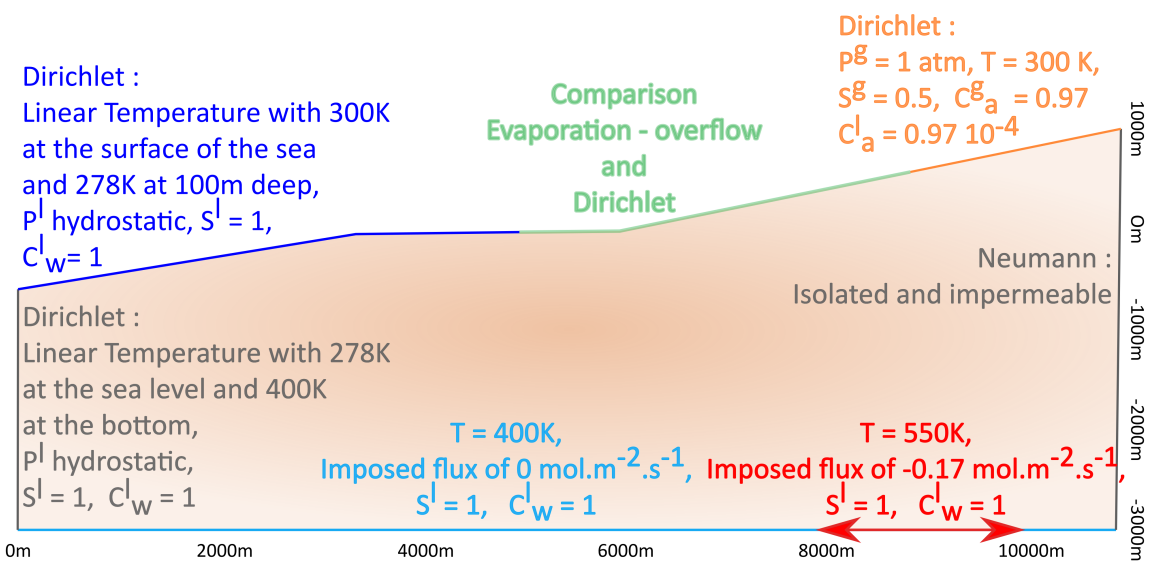

Fig. 1 Illustration of the 2D domain and the boundary conditions of the test case.

The convective molar and energy transfer layer thicknesses are fixed to $\delta_{m}=$ $\delta_{T}=10^{-1} \mathrm{~m}$. The far field atmospheric conditions are set to $C_{a, \infty}^{g, \text { atm }}=0.98, C_{w, \infty}^{g, a t m}=$ $0.02, T_{\infty}^{\text {atm }}=300 \mathrm{~K}$ and $P^{\mathrm{atm}}=1 \mathrm{~atm}$.

The solution obtained using our evaporation - overflow boundary condition is compared with the solution obtained using a Dirichlet boundary condition prescribing directly the gas saturation $S^{g}=1$, molar fractions $C_{a}^{g}=1, C_{w}^{g}=0$, pressure $P^{g}=1 \mathrm{~atm}$ and temperature $T=300 \mathrm{~K}$.
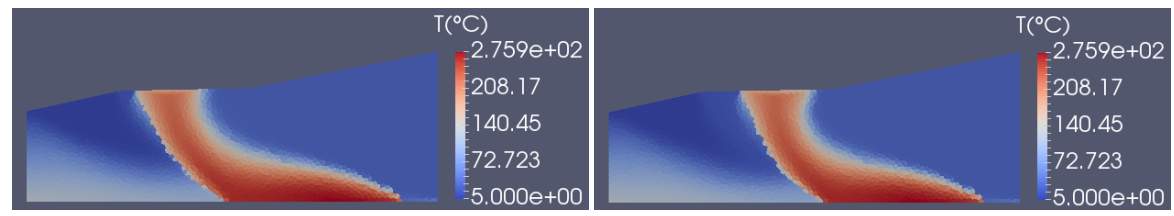

Fig. 2 Temperature at final time with the Evaporation - Overflow boundary condition (on the left) and with Dirichlet boundary condition (on the right).

We observe in figures 2 and 3 that the evaporation - overflow condition favours the exit of the hot liquid flux in the sea (located between $x=0 \mathrm{~m}$ and $x=5000 \mathrm{~m}$ ) and provides a better match with what happens in the Bouillante geothermal field. This 

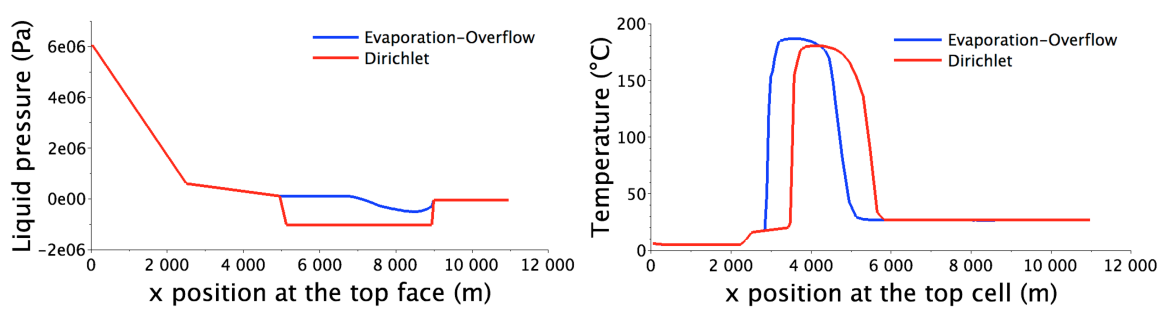

Fig. 3 Plots of the liquid pressure at the top boundary and of the temperature at the top cell at final time with the Evaporation - Overflow and Dirichlet boundary conditions.

can be explained by the lower liquid pressure $P^{l}=P^{a t m}-P_{c}(1)$ provided at the top boundary by the gas Dirichlet condition than the one provided by the evaporation overflow condition with in particular $P^{l}=P^{g}$ between say $x=5000 \mathrm{~m}$ and $x=6800$ $m$ as a result of the overflow condition.

Table 2 exhibits the good numerical behaviour of both test cases in terms of non linear and linear convergences. Note that the linear systems are solved using a GMRes iterative solver preconditioned by CPR-AMG.

Table 2 Numerical behaviour for both boundary conditions comparing the number of time steps $N_{\Delta t}$, the number of time step chops $N_{\text {chop }}$, the total number of Newton iterations $N_{\text {Newton }}$, the number of GMRes iterations by Newton iteration $N_{\text {GMRes }}$ and the CPU time.

\begin{tabular}{cccccc}
\hline & $N_{\Delta t}$ & $N_{\text {chop }}$ & $N_{\text {Newton }}$ & $N_{\text {GMRes }}$ & CPU time (s) \\
\hline Dirichlet boundary condition & 333 & 7 & 1835 & 22.3 & 436 \\
\hline Evaporation - Overflow boundary condition & 344 & 3 & 2072 & 21.1 & 404 \\
\hline
\end{tabular}

Acknowledgements We would like to thank the BRGM and the Provence-Alpes-Côte d'Azur Region for the co-funding of the PhD of Laurence Beaude as well as the support of the CHARMS ANR project (ANR-16-CE06-0009).

\section{References}

1. Ben Gharbia, I., Jaffré, J.: Gas phase appearance and disappearance as a problem with complementarity constraints. Mathematics and Computers in Simulation (2013)

2. Brenner, K., Groza, M., Jeannin, L., Masson, R., Pellerin, J.: Immiscible two-phase Darcy flow model accounting for vanishing and discontinuous capillary pressures: application to the flow in fractured porous media (2016). Working paper or preprint

3. Droniou, J.: Finite volume schemes for diffusion equations: Introduction to and review of modern methods. Mathematical Models and Methods in Applied Sciences 24(8), 1575-1619 (2014)

4. Lauser, A., Hager, C., Helmig, R., Wohlmuth, B.: A new approach for phase transitions in miscible multi-phase flow in porous media. Advances in Water Resources 34, 957-966 (2011)

5. Masson, R., Trenty, L., Zhang, Y.: Formulations of two phase liquid gas compositional Darcy flows with phase transitions. International Journal on Finite Volumes 11, 34 (2014)

6. O'Sullivan, M.J., Pruess, K., Lippmann, M.J.: Geothermal reservoir simulation: The state-ofpractice and emerging trends. Geothermics 30(4), 395-429 (2001)

7. Schmidt, E.: Properties of water and steam in S.I. units. In: . Springer-Verlag (1969) 